\title{
Effect of bronchial thermoplasty on airway distensibility
}

\author{
R.H. Brown*, W. Wizeman ${ }^{\#}$, C. Danek" and W. Mitzner*
}

\begin{abstract}
Recent studies have reported that the application of thermal energy delivered through a bronchoscope (bronchial thermoplasty) impairs the ability of airways to narrow in response to methacholine. How such altered smooth muscle affects the response of airways to lung inflation may have important clinical implications, particularly as it relates to the abnormal response of asthmatic subjects to lung inflation and deep inspiration. The aim of this study was to examine whether bronchial thermoplasty affected airway distension with lung inflation in relaxed and contracted airways.

A total of 230 airways were studied, ranging 2.5-15 mm, in six dogs. These airways were divided into two groups: an untreated (control) population and a bronchial thermoplasty-treated population.
\end{abstract}

Prior to treatment, the airway pressure-area curves in the two groups of airways were identical. In contrast, the relaxed and contracted airway pressure-area curves in treated airways were shifted upward at all points, showing increased airway area at both 3 and 5 weeks post-treatment.

In conclusion, these results show that reducing that amount of functional smooth muscle with bronchial thermoplasty leads to increased airway size in both relaxed and contracted states over a normal range of inflation pressures.

KEYWORDS: Airway smooth muscle, airway structure, asthma, lung mechanics

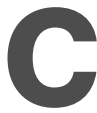
urrent studies have demonstrated that the application of thermal energy to the airway wall, termed bronchial thermoplasty, can impair the ability of airways to narrow in response to methacholine chloride (Mch) [1]. The technique was recently proposed as an innovative treatment to attenuate airway narrowing in asthma [2]. In another recent study, high-resolution computed tomography (HRCT) was used to accurately quantify the changes in airway area before and after a standard Mch aerosol challenge in canine airways treated with bronchial thermoplasty [3]. These study results showed that treated airways had significantly increased lumenal area at any dose of inhaled Mch when compared to untreated airways.

From previous work, the current authors have established that fully relaxed airways are quite distensible at low lung volume, but quickly reach a maximal size with no further distension up to total lung capacity (TLC). With smooth muscle tone, the airways do distend with increasing lung inflation, but maximal distension of even moderately constricted airways is not achieved, even at TLC [4]. This airway distension behaviour may be of more than academic interest, since the response to lung inflation and deep inspiration is known to be different in asthmatic subjects when compared to healthy individuals. After a deep inspiration in healthy subjects, airway contraction is reversed, whereas in asthmatic subjects, there is often no reversal or even a further constriction [5-8]. Thus, how an altered smooth muscle layer affects the response of airways to lung inflation may have important implications for this method of bronchial thermoplasty. To this end, this study examined whether bronchial thermoplasty would affect airway distension with lung inflation either when the airways were relaxed or had tone.

\section{METHODS}

This study examined the effect of treating multiple airways of six healthy mongrel dogs with bronchoscopically delivered radiofrequency energy. The study protocol was approved by The Johns Hopkins Animal Care and Use Committee (Baltimore, MD, USA). Six dogs, weighing $\sim 20 \mathrm{~kg}$, were anaesthetised with thiopental $\left(15 \mathrm{mg} \cdot \mathrm{kg}^{-1}\right.$ induction dose followed by $10 \mathrm{mg} \cdot \mathrm{kg}^{-1} \cdot \mathrm{h}^{-1}$ intravenous maintenance dose). After induction of anaesthesia, the dogs were paralysed with $0.5 \mathrm{mg} \cdot \mathrm{kg}^{-1}$ of succinylcholine, with occasional supplemental doses as required to ensure no respiratory motion during imaging. Following endotracheal intubation with an
AFFILIATIONS

*Johns Hopkins University,

Baltimore, MD, and

\#Asthmatx, Mountain View, CA, USA.

CORRESPONDENCE

W. Mitzner

Johns Hopkins University

Environmental Health Sciences

615 N. Wolfe Street

Baltimore

MD 21205

USA

Fax: 14109550299

E-mail:wmitzner@jhsph.edu

Received:

January 182005

Accepted after revision:

April 252005 
8.0-mm internal diameter endotracheal tube, the dogs were placed supine and their lungs were ventilated with room air with a volume-cycled ventilator (Harvard Apparatus, Millus, MA, USA) at a tidal volume of $15 \mathrm{~mL} \cdot \mathrm{kg}^{-1}$ and a rate of 18 breaths $\cdot \mathrm{min}^{-1}$. A stable depth of anaesthesia was maintained by monitoring heart rate changes and eyelash reflex.

\section{Treatment with the Alair System}

Treatments were performed throughout the targeted lung region in all accessible intra-pulmonary airways. The animals were treated with the Alair ${ }^{\circledR}$ System (Asthmatx, Inc., Mountain View, CA, USA) for bronchial thermoplasty, comprising a lowpower RF generator and a basket catheter with four electrodes. The RF generator supplied power using temperature feedback control, to maintain the target treatment setting for $10 \mathrm{~s}$ at each treatment site. Entire lung regions of all accessible intraparenchymal sites $\geqslant 2 \mathrm{~mm}$ in diameter and distal to the carina were treated with contiguous activations of the device. The left and right sides of the lungs were used to assign treatment and control regions in this study. One side of the lungs in each dog received treatments of all accessible and visible airways (quantifiable on HRCT) at a temperature setting of $75^{\circ} \mathrm{C}$. The allocation of treatment and control (untreated) lung side for each dog was selected at random, such that three dogs were assigned treatment to the left lung and three to the right. The untreated lung served as an internal control in each animal. Observers were blinded to treatment conditions for all HRCT measurements of airway area.

\section{Protocol}

Each dog served as its own control. The dogs were anaesthetised and ventilated as described above. On separate days, in random order, the dogs received a continuous intravenous infusion of methacholine $\left(67 \mu \mathrm{g} \cdot \mathrm{min}^{-1}\right.$; Sigma Chemical, St Louis, MO, USA), a dose previously demonstrated to decrease the size of the airways to $\sim 60 \%$ of baseline [4], or intravenous atropine $\left(0.2 \mathrm{mg} \cdot \mathrm{kg}^{-1}\right)$, a dose previously shown to completely block vagal tone in dogs [4]. To standardise lung volume history, prior to each measurement, the airway pressure was increased to $35 \mathrm{cmH}_{2} \mathrm{O}$, held for $5 \mathrm{~s}$ and then decreased and maintained at the designated airway pressure for the duration of each set of scans. At each pressure, HRCT scans were acquired to measure airway areas. Scans were acquired at airway pressures of $0,3,10$, 16 and $30 \mathrm{cmH}_{2} \mathrm{O}$. Between each experimental condition, the animals were ventilated normally. From these pressure-area curves, the specific airway compliance $\left(C_{s}\right)$ was calculated from the fractional changes in area with lung inflation from 0 to $10 \mathrm{cmH}_{2} \mathrm{O}$, as follows:

$$
C_{s}=\left(\left(\mathrm{A}_{10}-\mathrm{A}_{0}\right) / \mathrm{A}_{30}\right) /\left(\mathrm{P}_{10}-\mathrm{P}_{0}\right)
$$

Where A10 is the airway luminal area at $10 \mathrm{cmH}_{2} \mathrm{O}, \mathrm{A} 0$ is the airway luminal area at $0 \mathrm{cmH}_{2} \mathrm{O}, \mathrm{A} 30$ is the maximum airway luminal area at $30 \mathrm{cmH}_{2} \mathrm{O}, \mathrm{P} 10$ is the airway pressure at $10 \mathrm{~cm}$ $\mathrm{H}_{2} \mathrm{O}$ and $\mathrm{P} 0$ is the airway pressure at $0 \mathrm{cmH}_{2} \mathrm{O}$.

\section{Imaging and analysis of airways}

HRCT scans were obtained using a Somatom Volume Zoom scanner (Siemens, Iselin, NJ, USA), using a spiral mode to acquire $60 \mathrm{CT}$ images during an 8-s breath hold (apnoea) at $137 \mathrm{kVp}$ and $165 \mathrm{~mA}$. The images were reconstructed at a 1-mm slice thickness and a $512 \times 512$ matrix, using a $125-\mathrm{mm}$ field of view and a high spatial frequency (resolution) algorithm, which enhanced edge detection, at a window level of $-450 \mathrm{HU}$ and a window width of 1,350 HU. These settings have been shown to provide accurate measurement of lumenal size as small as $0.5 \mathrm{~mm}$ in diameter $[9,10]$. For repeated airway measurements in a given dog within each experimental protocol, adjacent anatomic landmarks, such as airway or vascular branching points, were defined and used to measure the airway size at the same anatomic cross sections. Using these landmarks, it was possible to match the location of each airway in the stacked series of CT images with that obtained from the bronchoscopic treatment location. The bronchoscopic location of each treated airway was determined by mapping the airway location with the procedure previously described in detail by DANEK et al. [1].

The HRCT images were analysed using the airway analysis module of the Volumetric Image and Display Analysis (VIDA) image analysis software package (Dept of Radiology, Division of Physiologic Imaging, University of Iowa, Iowa City, IA, USA), as previously described and validated [4,11]. The HRCT images were transferred to a UNIX-based Sun workstation. An initial isocontour was drawn within each airway lumen, and the software program then automatically located the perimeter of the airway lumen by sending out rays in a spoke-wheel fashion to a pre-designated pixel intensity level that defines the lumenal edge of the airway wall. Intra- and inter-observer accuracy and variability of the software program using this HRCT technique in phantoms, consisting of rigid tubes to measure known areas, has been previously shown by the authors $[9,11]$ and by others to be highly resistant to operator bias.

\section{Data analysis}

In each dog, 47-56 airways (range 2.5-15 $\mathrm{mm}$ in diameter after atropine at $0 \mathrm{cmH}_{2} \mathrm{O}$ ) were identified and measured under all conditions. The mean airway areas after atropine at $0 \mathrm{cmH}_{2} \mathrm{O}$ for treated and untreated airways were compared by unpaired t-test. To compare changes after treatment or Mch challenge in individual airways, $100 \%$ airway area was defined for each airway as that measured at the pre-treatment measurement time after atropine relaxation and at $30 \mathrm{cmH}_{2} \mathrm{O}$ (the initial maximal size). Subsequent airway lumenal area measurements are all expressed as a per cent of this initial maximal airway size. Airway responses to lung inflation were analysed separately for Mch and atropine challenges by generalised analysis of variance, with the airway luminal area (as per cent of maximum) the dependent variable, and the multiple airways per dog, the bronchial thermoplasty treatment and the timing of scanning relative to the treatment date the independent variables. Bonferroni corrections were made for multiple pairwise comparisons of means. Specific airway compliances were analysed separately for Mch and atropine challenges by one-way analysis of variance comparing treated and untreated airways for the three time points. Significance was considered if the p-value was $<0.05$.

\section{RESULTS}

\section{Pre-treatment data}

A total of 97 airways were treated and measured (range 7-21 airways per dog), with a mean relaxed airway size of $6.9 \mathrm{~mm}$ 
in diameter (range $2.5-15 \mathrm{~mm}$ in diameter). A total of 133 untreated airways over the same size range were identified and measured (range 22-39 airways per dog), with a mean relaxed airway size of $6.8 \mathrm{~mm}$ in diameter (range $2.5-15 \mathrm{~mm}$ in diameter). For airways without muscle tone, there was no significant difference between the mean size of the group of airways to be treated and those to be left untreated $(p=0.9)$. Prior to treatment, at functional residual capacity (FRC), the mean sizes of the subsequently treated and the untreated airways were not different either during Mch infusion $(16 \pm 1$ and $15 \pm 1 \%$ of their initial maximum size, respectively, $\mathrm{p}=0.18)$ or after atropine administration $(66 \pm 2$ and $64 \pm 1 \%$ of their initial maximum size, respectively, $\mathrm{p}=0.49)$.

\section{Responses to increasing pressure}

Increasing airway pressure caused an increase in airway size. After atropine relaxation, the airways increased significantly in size at each increase in airway pressure and reached a plateau at $16 \mathrm{cmH}_{2} \mathrm{O}$. At $10 \mathrm{cmH}_{2} \mathrm{O}$, the airway size was $95 \pm 2$ and $94 \pm 2 \%$ of their initial maximum size for the subsequently treated and the untreated airways, respectively $(\mathrm{p}<0.0001$ compared to the initial maximal size, fig. 1a). At $16 \mathrm{cmH}_{2} \mathrm{O}$, the airway size was $102 \pm 1(p=0.71)$ and $101 \pm 2 \%(p=0.33)$ of their respective initial maximum size for the subsequently treated and the untreated airways, respectively (fig. 1a). In addition, in these subsequently treated and untreated airways, there were no differences in the size of the airways at any airway pressure $(\mathrm{p}=0.88)$.

During Mch contraction, the airways remained narrowed until the airway pressure increased to $16 \mathrm{cmH}_{2} \mathrm{O}$, when the airway increased significantly in size to $24 \pm 1(p=0.0007)$ and $23 \pm 1 \%$ $(p<0.0001)$ of their initial maximum size for the subsequently treated and untreated airways, respectively (fig. 2a). At $30 \mathrm{cmH}_{2} \mathrm{O}$, the airways further significantly increased in size up to $46 \pm 3(\mathrm{p}<0.0001)$ and $42 \pm 2 \%(\mathrm{p}<0.0001)$ of their initial maximum size for the subsequently treated and the untreated airways, respectively (fig. 2a). In addition, in these subsequently treated and untreated airways, there were no differences in the size of the airways at any airway pressure $(\mathrm{p}=0.11)$.

The dose-response curves to airway pressure in treated airways after atropine administration were shifted upward at both $3(p<0.0001)$ and $5(p<0.0001)$ weeks post-treatment compared to pre-treatment. Likewise, the dose-response curves to airway pressure in Mch challenged treated airways
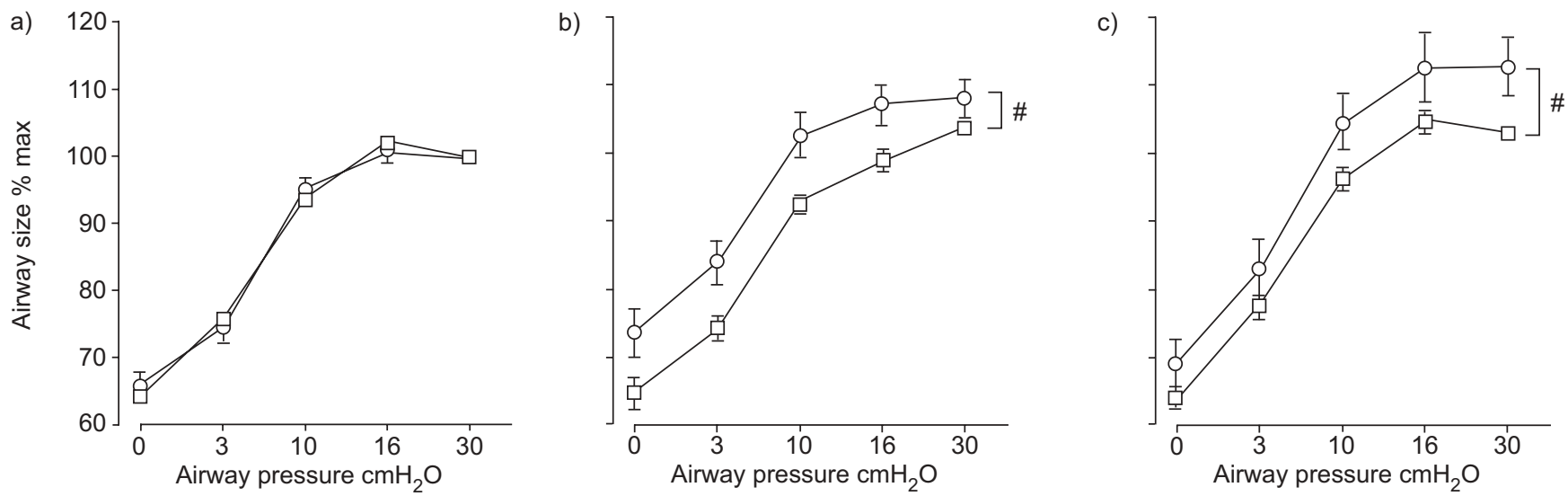

FIGURE 1. Airway size (mean \pm SEM) at baseline and at increasing airway pressure after atropine a) pre-treatment, and b) 3 weeks and c) 5 weeks post-treatment in the subsequently treated $(\bigcirc)$ and untreated $(\square)$ airways. ${ }^{*}: p<0.0001$.
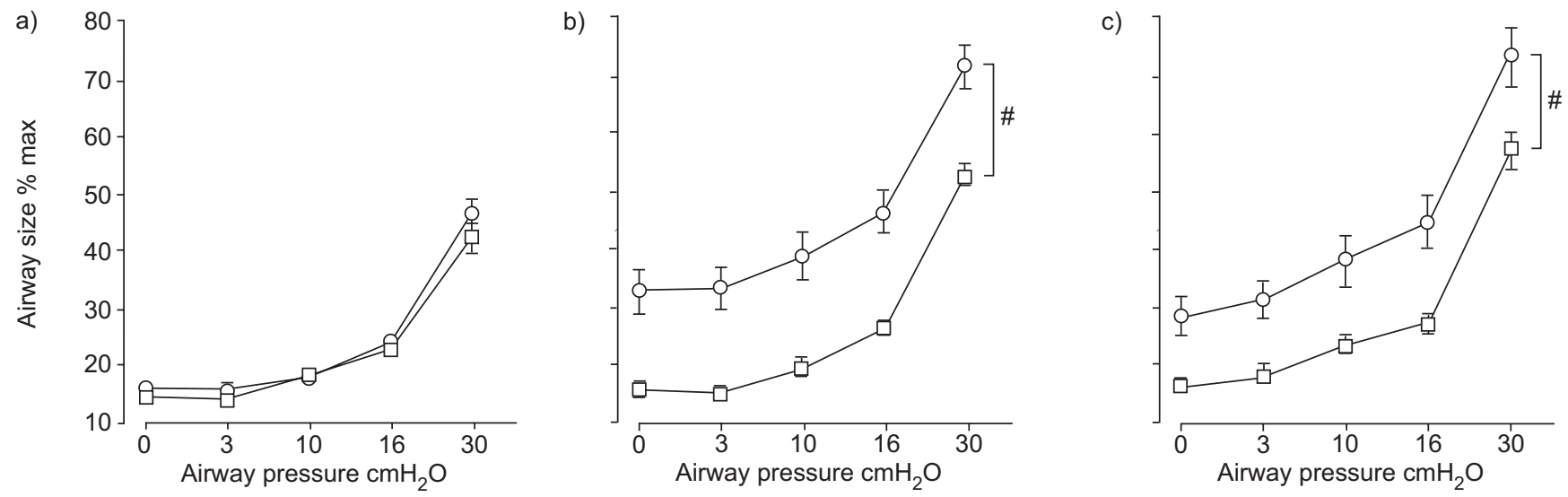

FIGURE 2. Airway size (mean \pm SEM) at baseline and at increasing airway pressure during methacholine chloride (Mch) infusion a) pre-treatment, and b) 3 weeks and c) 5 weeks post-treatment with Mch infusion in the subsequently treated $(\bigcirc)$ and untreated $(\square)$ airways. ${ }^{\#}: p<0.0001$. 
were shifted upward, indicating a significantly decreased responsiveness and less intrinsic structural tone at both 3 $(\mathrm{p}<0.0001)$ and $5(\mathrm{p}<0.0001)$ weeks post-treatment compared to pre-treatment. As there were no qualitative differences in the individual airway responses among dogs, in the subsequent figures we show the group means at each time point for treated and untreated airways.

\section{Responses at 3 weeks post-treatment}

Three weeks after bronchial thermoplasty, after atropine administration, there was a significant difference between the treated and the untreated airways $(p<0.0001$, fig. 1b). At $0 \mathrm{cmH}_{2} \mathrm{O}$, the mean size of the treated and the untreated airways was significantly different at $74 \pm 3$ and $65 \pm 2 \%$ of their initial maximum size respectively $(\mathrm{p}=0.02)$. At $30 \mathrm{cmH}_{2} \mathrm{O}$, the mean size of the treated airways was $108 \pm 3 \%$ of the initial maximal size $(\mathrm{p}=0.007)$, and that of the untreated airways was $104 \pm 2 \%$ of the initial maximum size $(p=0.17$, fig. $1 b)$. In addition, the treated airways reached a size plateau at the lower pressure of $10 \mathrm{cmH}_{2} \mathrm{O}(\mathrm{p}=0.20$ compared to airway size at $30 \mathrm{cmH}_{2} \mathrm{O}$ ).

Similarly, 3 weeks after bronchial thermoplasty, during Mch infusion, at every airway pressure there was also a significant difference between the treated and the untreated airways $\left(\mathrm{p}<0.0001\right.$, fig. $2 \mathrm{~b}$ ). At $0 \mathrm{cmH}_{2} \mathrm{O}$, the mean size of the treated and the untreated airways was significantly different at $33 \pm 4$ and $16 \pm 2 \%$ of their initial maximum size, respectively $(\mathrm{p}<0.0001)$. At $30 \mathrm{cmH}_{2} \mathrm{O}$, the mean size of the treated and the untreated airways remained significantly different at $71 \pm 2$ and $52 \pm 2 \%$ of their maximum sizes, respectively $(\mathrm{p}<0.0001$, fig. $2 b)$.

\section{Responses at 5 weeks post-treatment}

Five weeks after bronchial thermoplasty, after atropine administration, there was a significant difference between the treated and the untreated airways $(p<0.0001$, fig. $1 c)$. At $0 \mathrm{cmH}_{2} \mathrm{O}$, the mean size of the treated and the untreated airways was not significantly different at $69 \pm 3$ and $64 \pm 2 \%$ of their initial maximum size, respectively $(\mathrm{p}=0.15)$. At $30 \mathrm{cmH}_{2} \mathrm{O}$, the mean size of the treated airways was $112 \pm 4 \%$ of the initial maximal size $(\mathrm{p}=0.005)$ and that of the untreated airways was $103 \pm 1 \%$ of the initial maximum size $(\mathrm{p}=0.03$, fig. 1c). As at 3 weeks, the treated airways reached their maximum size plateau at the lower pressure of $10 \mathrm{cmH}_{2} \mathrm{O}$ $\left(\mathrm{p}=0.17\right.$ compared to airway size at $\left.30 \mathrm{cmH}_{2} \mathrm{O}\right)$.

Similarly, 5 weeks after bronchial thermoplasty, during Mch infusion, at every airway pressure, there was also a significant difference between the treated and the untreated airways $(\mathrm{p}<0.0001$, fig. $2 \mathrm{c})$. At $0 \mathrm{cmH}_{2} \mathrm{O}$, the mean size of the treated and the untreated airways was significantly different at $28 \pm 3$ and $16 \pm 1 \%$ of their initial maximum size, respectively $(\mathrm{p}<0.0001)$. At $30 \mathrm{cmH}_{2} \mathrm{O}$, the mean size of the treated and the untreated airways remained significantly different at $73 \pm 5$ and $57 \pm 3 \%$ of their initial maximum size, respectively $(\mathrm{p}=0.004$, fig. 2c).

\section{Compliance data}

Table 1 shows the specific compliance for the different times and treatment groups. As expected, there were significant decreases in compliance with Mch in both treated and

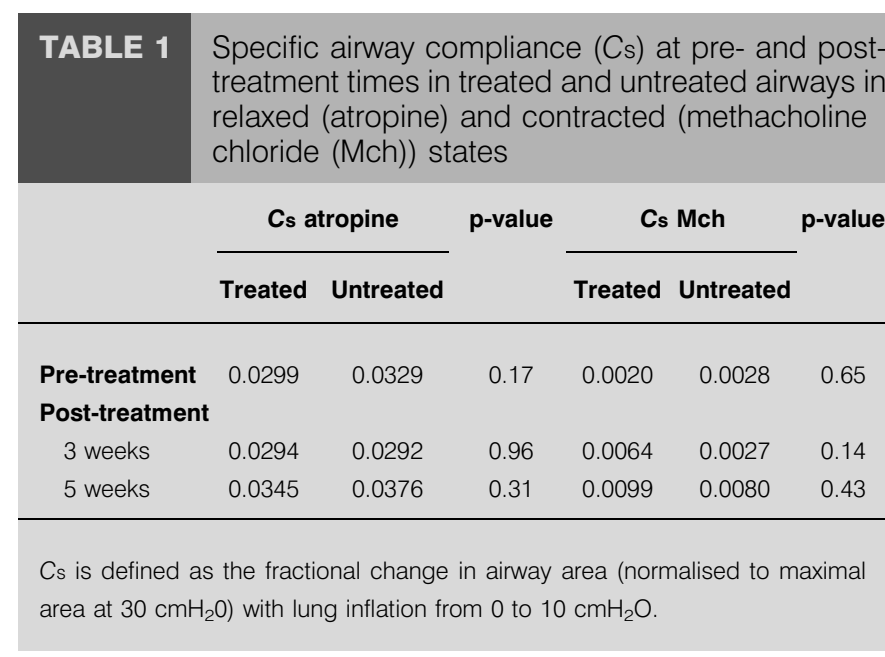

untreated airways. However, there were no differences between treated and untreated airways at any time point.

\section{DISCUSSION}

The results presented in this study show that bronchial thermoplasty can significantly increase airway dimensions at any degree of lung inflation for up to 5 weeks following treatment. Furthermore, the improvement in distension occurred whether the airway smooth muscle was relaxed or contracted with Mch. While the animals were not followed beyond 5 weeks, in the previous work by DANEK et al. [1], dogs with a similar treatment were followed for up to 3 yrs following treatment. They found that the effect of treatment on airway responsiveness to Mch was maintained over this extended time period. Thus, it can be presumed that the increased distensibility observed in this study would also be stable for extended periods.

In a separate study by the present authors, where CT imaging was used to quantify the magnitude of decreased responsiveness of airway smooth muscle after bronchial thermoplasty in these treated dogs, animals were only studied at FRC (airway pressure $0 \mathrm{cmH}_{2} \mathrm{O}$ ). Consistent with this previous report, the present study found that the treated airways at $0 \mathrm{cmH}_{2} \mathrm{O}$ during Mch infusion were significantly larger after treatment $(33 \pm 4$ at 3 weeks and $28 \pm 3 \%$ at 5 weeks versus $16 \pm 2$ and $16 \pm 1 \%$ pre-treatment of their initial maximum size, respectively). Thus, prior to lung inflation, the treated airways were less responsive to the same dose of Mch, while the response of untreated airways remained unchanged over the 5-week interval $(16 \pm 2$ at 3 weeks and $16 \pm 1 \%$ at 5 weeks versus $15 \pm 1 \%$ pre-treatment of their initial maximum size, respectively). These results show that the previously reported reduced Mch responsiveness, performed only at FRC [3], cannot be eliminated at higher lung volumes.

In airways with cholinergic muscle tone abolished with atropine, it was found that the treated airways had increased size at all pressures. The maximum airway lumenal size increased slightly from $100 \%$ pre-treatment, to $108 \pm 3 \%$ at 3 weeks $(p=0.04)$ and $112 \pm 4 \%$ at 5 weeks $(p=0.002)$ compared to their pre-treatment initial maximum size. Untreated airways maintained a consistent maximum size at $30 \mathrm{cmH}_{2} \mathrm{O}$ over the 
5-week interval (104 $\pm 2 \%$ at 3 weeks and $103 \pm 1 \%$ at 5 weeks). These observations show that the airways are not only larger during smooth muscle constriction, but also even without muscle tone. Although it is possible that there was still some noncholinergic tone after the large-dose atropine, previous work in the dog model has showed that this is too small to detect with the imaging methods used [4].

It is neither conventional nor easy to calculate compliance or distensibility from such nonlinear pressure-area curves as commonly shown by airways. As a gross but interpretable index, the fractional increase in airway area over the pressure range from 0 to $10 \mathrm{cmH}_{2} \mathrm{O}$ was assessed. This specific airway compliance is analogous the specific compliance of the lung as assessed in human pulmonary function laboratories [12]. The data shown in table 1 demonstrates that, as expected, there is significantly decreased airway compliance with Mch challenge. However, compared to untreated airways, bronchial thermoplasty did not alter the airway compliance at either the 3- or 5-week time point.

Consistent with previous work examining the pressure-area curves in canine airways [4], the relaxed airways were found to be quite distensible at pressures $<10 \mathrm{cmH}_{2} \mathrm{O}$ and quite rigid above this pressure. Similarly, with airway contraction, this previous work also showed that the airways at low pressures could be quite stiff, only enlarging at the highest pressures. In the present study, similar behaviour was found in both treated and untreated airways. In addition, the pressure above which the relaxed airways become nondistensible was not altered by treatment. However, the finding that the relaxed airways are slightly larger in treated airways without smooth muscle tone could not have been predicted from this previous work, nor could it have been detected with the direct visualisation approach originally used by DANEK et al. [1] in the original description of bronchial thermoplasty. DANEK et al. [1] only examined airways with normal baseline tone and did not make any attempt to abolish tone. However, they did examine treated and untreated airways histologically at various posttreatment times. In the current work, the amount of smooth muscle damage was not assessed histologically, but, since the treatment protocols were essentially identical, it was assumed that the histological changes were similar to those shown by DANEK et al. [1]. This histology showed that the only observable changes were a reduction in the amount of smooth muscle in the treated airway walls. Smooth muscle was still present, but there was a decrease in the fraction of the airway perimeter that had identifiable smooth cells. This being the case, how can the findings in this present work be interpreted?

Perhaps the most likely cause of the increase in maximum airway lumenal area relates to the absolute loss or alteration of smooth muscle mass. Even with no tone, a decreased amount of total muscle mass around the airway might allow the airway to reach a slightly larger size. Although DANEK et al. [1] reported observations of reduced airway smooth muscle following bronchial thermoplasty, morphometric quantification of the amount of smooth muscle damage by the bronchial thermoplasty treatment has not been done, and is beyond the scope of the present work. Another possibility is that there was some structural alteration to other (nonmuscle) components in the airways, such as collagen or other fibrous elements that were not detected by DANEK, et al. [1]. Even slight alterations to these structural elements in the airway wall from the bronchial thermoplasty could contribute to a slight dilation of the relaxed airways even without a reduction in baseline airway smooth muscle tone.

Although there was probably acute damage to other airway structures, such as nerves or vessels, from bronchial thermoplasty, it is felt that it is most likely the direct action on airway smooth muscle that caused the attenuation of airway constriction. Epithelium, blood vessels and nerves all have a good capacity for regeneration, a fact supported by direct observations of the airways. During the bronchoscopy at the time of treatment, some minor airway epithelial damage was observed immediately after treatment. However, CT scans were not performed on the animals immediately after treatment. The first CT measurements post-treatment were not done until at least 2 weeks after the treatment, and by this time there were no gross changes in the appearance of airway or parenchymal structures on CT scans.

GUNST et al. [13] have suggested that if the smooth muscle contraction is high enough, physiological increases in transpulmonary pressure might be insufficient to cause any increase in airway size. As was seen in the untreated airways during the Mch infusion, even at $30 \mathrm{cmH}_{2} \mathrm{O}$, the airway only reached $50 \%$ of the initial maximum size. Moreover, the treated airways were able to increase to $\sim 75 \%$ of their initial maximum size even during infusion of Mch. Thus, if the size at the maximal distending pressure is simply looked at, the treated airways with smooth muscle tone are clearly more distensible than the untreated airways. This is consistent with there being a decreased amount of functional smooth muscle in the airway [1], an observation that relates to the speculation of GUNST et al. [13], and has potential clinical implications. In clinical asthma, the intrinsic forces of constriction may be greater than that which lung inflation can overcome. Such a speculation is consistent with several observations showing that a deep inspiration does not relieve bronchoconstriction in asthmatic patients [8, 14-18]. A decreased amount of smooth muscle in the bronchial thermoplasty-treated airways should allow contracted airways to be more easily distended with a deep inspiration. The fact that the airway compliance in treated airways was not altered and that the maximal size of these airways was slightly increased, leads to the speculation that if one were to treat asthmatic airways, they might then be expected to behave more like the airways in healthy individuals in terms of their response to deep inspiration. One potentially relevant comment arises from a speculation made in a brief review by DRAZEN [19]. It was stated that with no smooth muscle tone in an airway, the wall would become more compliant, and this might lead to a decreased maximal expiratory flow. Although theoretically possible, there are several facts that would make such a scenario highly unlikely. First, in the wave speed formula for maximal flow, the actual airway area is a much greater determinant (varying to the 3rd power) than the airway compliance. Secondly, in the present studies no change was found in the compliance at lower lung volumes, only a shift in the pressure-area curve position. Thus, the increased area could only lead to increased maximal flow. Finally, there is no experimental evidence showing that relaxation of smooth muscle in parenchymal airways leads to 
a decreased maximal flow. If this was even a rare occurrence, treatment of acute bronchospasm with bronchodilators would not be such a commonly recommended therapy.

In conclusion, it has been shown here that reducing the amount of functional smooth muscle with bronchial thermoplasty leads to increased airway size in both the relaxed and contracted states over a normal range of inflation pressures. If similar effects were found in human airways treated with this procedure, then this increased airway size would probably be a beneficial effect in diseases with excessive airway narrowing [2].

\section{REFERENCES}

1 Danek CJ, Lombard CM, Dungworth DL, et al. Reduction in airway hyperresponsiveness to methacholine by the application of RF energy in dogs. J Appl Physiol 2004; 97: 1946-1953.

2 Cox PG, Miller J, Mitzner W, Leff AR. Radiofrequency ablation of airway smooth muscle for sustained treatment of asthma: preliminary investigations. Eur Respir J 2004; 24: 659-663.

3 Brown R, Wizeman W, Danek C, Mitzner W. In vivo evaluation of the effectiveness of bronchial thermoplasty with computed tomography. J Appl Physiol 2005; (In press).

4 Brown RH, Mitzner W. Effect of lung inflation and airway muscle tone on airway diameter in vivo. Journal Appl Physiol 1996; 80: 1581-1588.

5 Brown RH, Mitzner W. Airway response to deep inspiration: role of inflation pressure. J Appl Physiol 2001; 91: 2574-2578.

6 Wheatley JR, Pare PD, Engel LA. Reversibility of induced bronchoconstriction by deep inspiration in asthmatic and normal subjects. Eur Respir J 1989; 2: 331-339.

7 Gayrard P, Orehek J, Grimaud C, Charpin J. Bronchoconstrictor effects of a deep inspiration in patients with asthma. Am Rev Respir Dis 1975; 111: 433-439.

8 Fish JE, Ankin MG, Kelly JF, Peterman VI. Regulation of bronchomotor tone by lung inflation in asthmatic and nonasthmatic subjects. J Appl Physiol 1981; 50: 1079-1086.

9 Herold CJ, Brown RH, Mitzner W, Links JM, Hirshman CA, Zerhouni EA. Assessment of pulmonary airway reactivity with high-resolution CT. Radiology 1991; 181: 369-374.

10 Wood SA, Zerhouni EA, Hoford JD, Hoffman EA, Mitzner W. Measurement of three-dimensional lung tree structures by using computed tomography. J Appl Physiol 1995; 79: 1687-1697.

11 Amirav I, Kramer SS, Grunstein MM, Hoffman EA. Assessment of methacholine-induced airway constriction by ultrafast high-resolution computed tomography. J Appl Physiol 1993; 75: 2239-2250.

12 Stubbing DG, Pengelly LD, Morse JL, Jones NL. Pulmonary mechanics during exercise in normal males. J Appl Physiol 1980; 49: 506-510.

13 Gunst SJ, Stropp JQ, Service J. Mechanical modulation of pressure-volume characteristics of contracted canine airways in vitro. J Appl Physiol 1990; 68: 2223-2229.

14 Beaupre A, Orehek J. Factors influencing the bronchodilator effect of a deep inspiration in asthmatic patients with provoked bronchoconstriction. Thorax 1982; 37: 124-128.

15 Beaupre A, Badier M, Delpierre S, Orehek J, Grimaud C. Airway responses of asthmatics to carbachol and to deep inspiration. Eur J Respir Dis 1983; 64: 108-112.

16 Fish JE, Peterman VI, Cugell D. Effect of deep inspiration on airway conductance in subjects with allergic rhinitis and allergic asthma. J Allergy Clin Immunol 1977; 60: 41-46.

17 Orehek J, Charpin D, Velardocchio JM, Grimaud C. Bronchomotor effect of bronchoconstriction-induced deep inspirations in asthmatics. Am Rev Respir Dis 1980; 121: 297-305.

18 Orehek J, Nicoli MM, Delpierre S, Beaupre A. Influence of the previous deep inspiration on the spirometric measurement of provoked bronchoconstriction in asthma. Am Rev Respir Dis 1981; 123: 269-272.

19 Drazen JM. Asthma and the human genome project: summary of the 45th Annual Thomas L. Petty Aspen Lung Conference. Chest 2003; 123: Suppl. 3, 447S-449S. 